\title{
Avaliação do Desmatamento no Estado de Rondônia entre 2001 e 2011
}

\author{
Valderli Jorge Piontekowski ${ }^{1}$, Eraldo Aparecido Trondoli Matricardi ${ }^{1}$, \\ Marcos Antonio Pedlowski², Luis Cláudio Fernandes ${ }^{3}$
}

\author{
${ }^{1}$ Departamento de Engenharia Florestal, Universidade de Brasília - UnB, Brasília/DF, Brasil \\ ${ }^{2}$ Centro de Ciências do Homem, Universidade Estadual do Norte Fluminense Darcy Ribeiro - UENF, \\ Campos dos Goytacazes/RJ, Brasil \\ ${ }^{3}$ Coordenadoria de Geociências, Secretaria de Estado do Desenvolvimento Ambiental de Rondônia - SEDAM, \\ Porto Velho/RO, Brasil
}

\begin{abstract}
RESUMO
No presente estudo, foi avaliada a acurácia dos mapeamentos do desmatamento conduzidos pelo Programa de Monitoramento do Desflorestamento na Amazônia (PRODES) do Instituto Nacional de Pesquisas Espaciais (INPE) e pela Secretaria de Estado do Desenvolvimento Ambiental (SEDAM), para o Estado de Rondônia, no período entre 2001 a 2011, utilizando imagens Landsat 5 TM. Com base nos resultados deste estudo, os mapeamentos conduzidos pela SEDAM e pelo PRODES-INPE apresentaram coeficientes Kappa similares, estimados em 0,89 e 0,87 , respectivamente. Os dados do desmatamento dos sistemas PRODES e SEDAM revelaram um decréscimo nas taxas de desmatamento em todo o Estado de Rondônia no período de análise, embora os desmatamentos ilegais dentro de áreas protegidas tenham aumentado cerca de $400 \%$ entre 2002 e 2011. Assumindo-se esta tendência de desmatamento da última década, é possível afirmar que as terras indígenas e unidades de conservação (UC) em Rondônia serão os principais alvos de desmatamento e destruição nos próximos anos.
\end{abstract}

Palavras-chave: sensoriamento remoto, mudanças no uso e na cobertura da terra, Floresta Amazônica.

\section{Deforestation Assessment in the State of Rondônia between 2001 and 2011}

\begin{abstract}
The goal of this study was to assess the accuracy of deforestation maps produced by the National Institute for Spatial Research (INPE) and the Rondônia State Secretariat of Environmental Development (SEDAM) for the state of Rondônia between 2001 and 2011. Our results show that the deforestation mappings conducted by SEDAM and PRODES-INPE present similar Kappa coefficients, which were estimated at 0.89 and 0.87 , respectively. The deforestation datasets prepared by PRODES and SEDAM show a decrease in deforestation rates in Rondônia state in the study period. However, there was a significant increase in illegal deforestation (approximately $400 \%$ within the protected areas) in this period. Based on the deforestation trend observed in the past decade, we can affirm that forests inside protected areas are the main targets for deforestation in Rondônia state.
\end{abstract}

Keywords: remote sensing, land use and land cover changes, Amazon Forest. 


\section{INTRODUÇÃO}

O Estado de Rondônia apresenta um histórico de ocupação marcado por políticas públicas desenvolvimentistas e de ocupação territorial baseada na remoção da floresta nativa. Estas políticas tiveram um forte impulso na década de 1970, com a implantação de projetos de assentamentos conduzidos pelo Instituto de Colonização e Reforma Agrária (INCRA) (Brandão \& Souza, 2006; Tourneau \& Bursztyn, 2010). Os assentamentos foram responsáveis por promover um grande fluxo migratório de diferentes partes do território brasileiro para dentro de Rondônia, servindo assim como elemento indutor para a expansão da fronteira agropecuária. Ao mesmo tempo, este influxo provocou mudanças expressivas na paisagem natural, com a substituição de florestas nativas por agricultura (temporária e permanente) e pecuária bovina (Tourneau \& Bursztyn, 2010).

Com isso, a compreensão da dinâmica do desmatamento e dos processos de ocupação via integração de análises técnicas e científicas se tornou elemento de forte interesse para a comunidade científica, o que resultou num grande número de pesquisas. Para tais estudos e análises, o uso de dados e técnicas de Sensoriamento Remoto (SR) e Sistema de Informações Geográficas (SIG) passou a ser considerado fundamental e indispensável na gestão de recursos naturais (Becker, 2002) e de conflitos de uso da terra (Gasparini et al., 2013).

A partir de 1988, o Instituto Nacional de Pesquisas Espaciais (INPE) passou a mapear de forma sistemática o desmatamento na Amazônia Legal através do Programa de Cálculo do Desflorestamento da Amazônia (PRODES). O PRODES utiliza imagens óticas obtidas pelo sensor Thematic Mapper (TM), que fica a bordo do satélite LANDSAT (Land Remote Sensing Satellite) (Shimabukuro \& Smith, 1991).

A partir de dados gerados com o PRODES, passaram a ser conhecidas as taxas anuais do desmatamento por corte raso da floresta, ou seja, quando havia conversão de floresta nativa para fins agrícolas e para o estabelecimento da atividade pecuária.

O governo do Estado de Rondônia também passou a realizar o monitoramento do desmatamento a partir da criação da Secretaria de Estado do Desenvolvimento Ambiental (SEDAM). A SEDAM iniciou os trabalhos de monitoramento do desmatamento ainda na década de 1990, a partir da estruturação de um laboratório de sensoriamento remoto e cartografia. Os dados gerados pela SEDAM também foram baseados no uso de imagens do satélite LANDSAT 5 TM e são utilizados, dentre outros fins, para o licenciamento ambiental em Rondônia.

Um aspecto particular em relação aos estudos conduzidos pelo PRODES e pela SEDAM se refere ao seguinte fato: quanto mais apurados e precisos forem os dados obtidos por mapeamentos, melhores serão as aplicações para estudos e análises, bem como para o gerenciamento dos recursos disponíveis na região. Estes mapeamentos também são ferramentas importantes para a avaliação da evolução da dinâmica da paisagem, especificamente no que se refere à manutenção de sua integridade.

No processo de definição de um modelo de qualidade, é indispensável a determinação dos seus parâmetros, os quais consideram a acurácia dos dados em termos de posição espacial, atributos e atualidade (Lingnau \& Antunes, 2003). Lingnau \& Antunes (2003) afirmam que as fontes de erros em dados digitais são diversas e estes podem ser causados pelo observador ou pelos equipamentos de medição que estão sendo utilizados. Os erros geralmente são classificados em grosseiros, sistemáticos e aleatórios (Thapa \& Bossler, 1992).

Nas imagens classificadas, é comum o erro posicional (planimétrico), erro temático, que consiste na incerteza de conteúdos e atributos provenientes da classificação digital, e o erro temporal, que é proveniente da desatualização da imagem (Lingnau \& Antunes, 2003). Deste modo, nos procedimentos de classificação digital de imagens, é relevante a avaliação do desempenho do classificador utilizado. Nesse caso, é desejável utilizar a avaliação da acurácia, que consiste na obtenção de alguma medida quantitativa do grau de acerto ou de erro alcançado em processos de classificação digital de imagens ou em mapeamentos, para orientar os usuários acerca da confiabilidade dos dados obtidos. A avaliação da acurácia é considerada crítica em todo tipo de mapeamento (Powell et al., 2004) e, por 
isso, os mapas obtidos a partir da classificação digital possuem um valor muito limitado se sua acurácia ou a sua precisão não forem apresentadas (Lingnau \& Antunes, 2003).

O presente estudo buscou avaliar a acurácia dos mapeamentos do desmatamento conduzidos pelo INPE e pela SEDAM, envolvendo diferentes técnicas de mapeamento de desmatamento. Os resultados deste estudo contribuem para a validação dos sistemas de monitoramento do desmatamento em Rondônia, apresentando as respectivas acurácias dos mapeamentos considerados, as diferenças das taxas de desmatamento e a localização dos mesmos em relação às áreas protegidas no Estado.

\section{MATERIAL E MÉTODOS}

A área do presente estudo envolveu todo o território do Estado de Rondônia, localizado entre os meridianos $66^{\circ} 36^{\prime} 49^{\prime \prime}$ e $60^{\circ} 43^{\prime} 17^{\prime \prime}$ de longitude Oeste e os paralelos $7^{\circ} 58^{\prime} 33^{\prime \prime}$ e $13^{\circ} 41^{\prime} 57^{\prime \prime}$ de latitude Sul. A área total de Rondônia é de aproximadamente
23,7 milhões de hectares, representando 4,7\% da Amazônia Legal (Figura 1).

As áreas protegidas ocupam aproximadamente $38 \%$ da área total do Estado de Rondônia, alcançando um total de 81 unidades, sendo 20 Terras Indígenas (TI), 23 Áreas de Proteção Integral (API) e 38 Áreas de Uso Sustentável (AUS) (Rondônia, 2007).

A classificação climática do Estado de Rondônia segundo Köppen é do tipo Aw, tropical chuvoso, com temperaturas médias anuais de $25,5^{\circ} \mathrm{C}$, máxima de $31,5{ }^{\circ} \mathrm{C}$ e mínima de $20,7{ }^{\circ} \mathrm{C}$, uma estação relativamente seca durante o ano e uma chuvosa, com chuvas marcadas entre os meses de setembro e maio, o que implica num total pluviométrico anual que excede os $2.000 \mathrm{~mm}$ (Brasil, 1978).

Os principais solos identificados em Rondônia são os Aluviais, Cambissolos, Glei, Hidromórficos, Concrecionários, Latossolos, Orgânicos, Podzólicos, Plintossolos, Areias, Litólicos, Terras Roxas e Planossolos (Rondônia, 2007). As principais formações vegetais incluem: Floresta Estacional Semidecidual, Floresta Ombrófila Aberta, Floresta

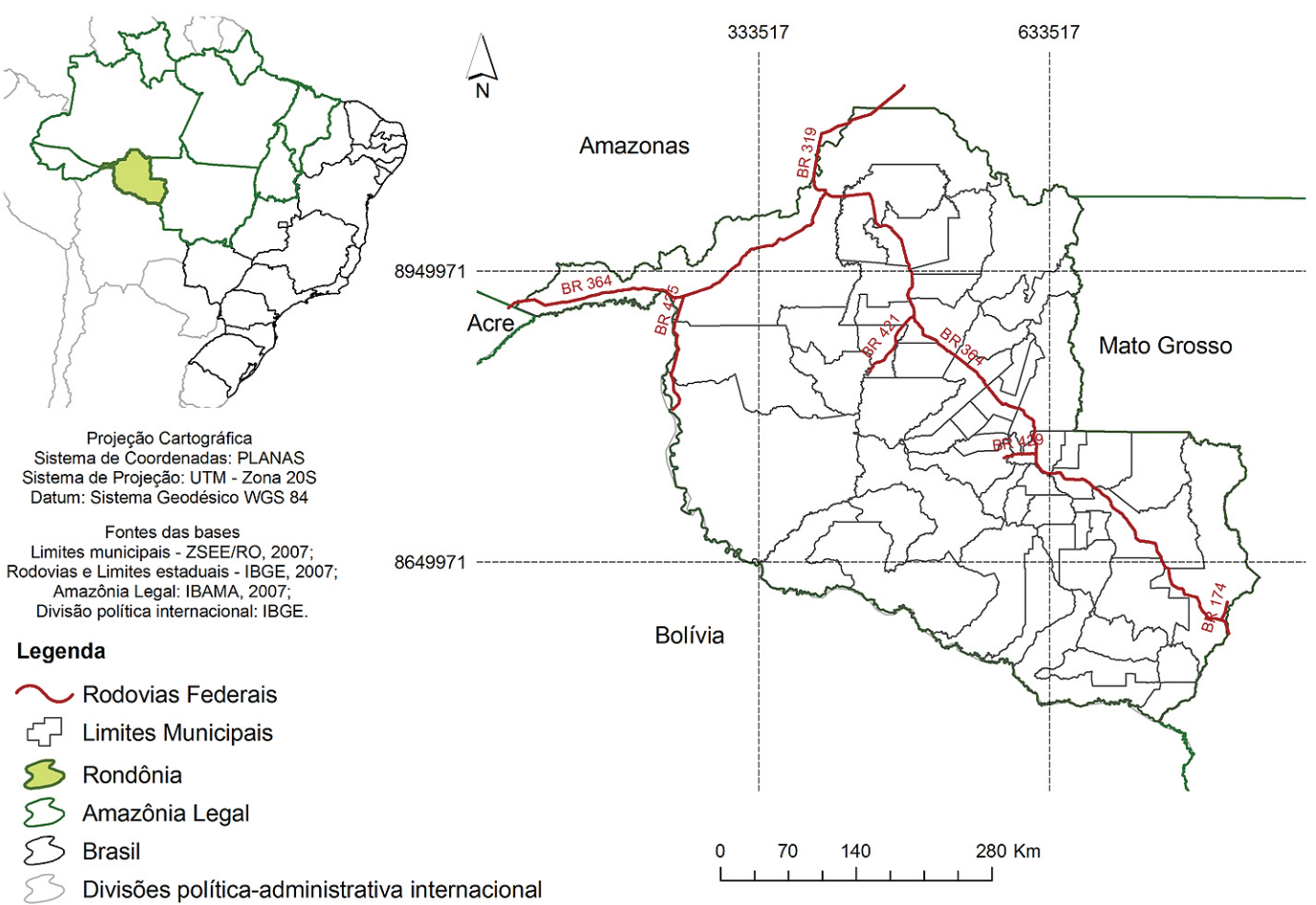

Figura 1. Localização do Estado de Rondônia no Brasil.

Figure 1. The state of Rondônia location in Brazil. 
Ombrófila Densa, Savana e as Formações Pioneiras de Influência Fluvial (Brasil, 1978).

Os dados temáticos (limite estadual, limites municipais e áreas protegidas) em formato vetorial utilizados neste trabalho foram produzidos pelo projeto de Zoneamento Socioeconômico Ecológico do Estado de Rondônia - ZSEE, em escala de 1:250.000 e sistema de coordenadas UTM, fuso UTM 20S, South America Datum (SAD), 1969. O aplicativo computacional utilizado para manipulação e geração dos resultados foi o software ArcMap ${ }^{\circledR}$, que é a aplicação central do sistema ArcGIS ${ }^{\circledast}$, versão 10.1.

Os dados de desmatamento de Rondônia que são utilizados neste estudo foram produzidos pela SEDAM e pelo PRODES para o período de 2001 a 2011. O mapeamento do desmatamento no Estado de Rondônia que foi elaborado pela SEDAM é baseado em interpretação visual de imagens do satélite LANDSAT 5 TM. Neste caso, os polígonos de novos desmatamentos foram digitalizados na tela de computador e os dados foram armazenados e disponibilizados em formato vetorial shapefile, envolvendo as classes anuais de desmatamento e a floresta nativa remanescente. Os dados do desmatamento do PRODES foram disponibilizados e adquiridos no sítio da internet do INPE (http://www. inpe.br/). O PRODES utiliza imagens do satélite LANDSAT 5 TM e distribui seus dados em formatos vetorial (shape e spring) e matricial (geotiff).

O PRODES conduz o mapeamento do desmatamento na Amazônia utilizando uma metodologia própria, baseada em frações de imagens (sombra, solo e vegetação) derivadas da Análise Linear de Mistura Espectral de imagens do LANDSAT 5 TM, seguida pela segmentação em campos homogêneos das imagens das frações solo e de sombra, e na aplicação de classificação não supervisionada para mapear as classes de uso da terra de interesse (desmatamento do ano, florestas remanescentes, etc.) (Câmara et al., 2006). Originalmente, os dados do PRODES contemplam sete classes: 'Floresta', 'Desflorestamento até 1997', 'Incremento do desmatamento' anual (de 2000 a 2011), 'Não Floresta' (outras formações vegetais, como o Cerrado), 'Nuvens,' 'Hidrografia' e 'Resíduo' (desmatamentos não detectados ocorridos em anos anteriores). Para efeito das análises no presente estudo, as classes foram agrupadas em 'Floresta', 'Hidrografia', 'Nuvens' e 'Não Floresta'. As classes 'Desflorestamento até 1997', 'Incremento anual do desmatamento' (mapeado a cada ano), e 'Resíduos' do desmatamento foram agrupadas como 'Desmatamento'. A classe 'Resíduo' foi incorporada como 'Desmatamento' no ano imediatamente anterior ao do ano mapeado. As classes 'Não Floresta', 'Hidrografia' e 'Nuvens' não foram consideradas nesta análise.

Para análise da acurácia dos dados de desmatamento, foram utilizadas neste estudo duas cenas (1542 e 1913) de imagens SPOT 5, localizadas no Estado de Rondônia (Figura 2), em formato de Números Digitais (ND), resolução radiométrica 8 bits, bandas multiespectrais 1, 2, 3 e 4 fusionadas com a banda pancromática do sensor HRG (High Resolution Geometrical), o que possibilitou a geração das respectivas cenas SPOT em resolução espacial de 2,6 metros.

A escolha destas duas cenas foi baseada na sua disponibilidade e na localização no território rondoniense, envolvendo regiões de ocupações mais antigas (cena 1913) com menores taxas atuais de desmatamento, e regiões de ocupações mais recentes ou novas fronteiras de ocupação (cena 1542), nas quais se concentram as mais altas taxas atuais de desmatamento no Estado. As imagens selecionadas foram adquiridas no ano de 2010, assim como os dados de desmatamento mapeados pela SEDAM e pelo PRODES-INPE.

Nas duas cenas SPOT, 400 pontos foram aleatorizados para a checagem da acurácia dos mapeamentos (Figura 2). Na sequência, os pontos aleatorizados foram checados sobre as imagens SPOT e comparados com as classes definidas pelo PRODES-INPE e pela SEDAM. Após a verificação do quantitativo de pontos correspondentes a cada classe (desmatado e não desmatado), estes foram inseridos numa matriz de confusão (erro), comparando a referência (imagens SPOT) e a classificação realizada pelo PRODES-INPE e pela SEDAM.

A partir da utilização da matriz de confusão, foram então estimados a exatidão global (Equação 1) (Congalton \& Mead, 1986) e o coeficiente Kappa (Equação 2) (Landis \& Koch, 1977). 


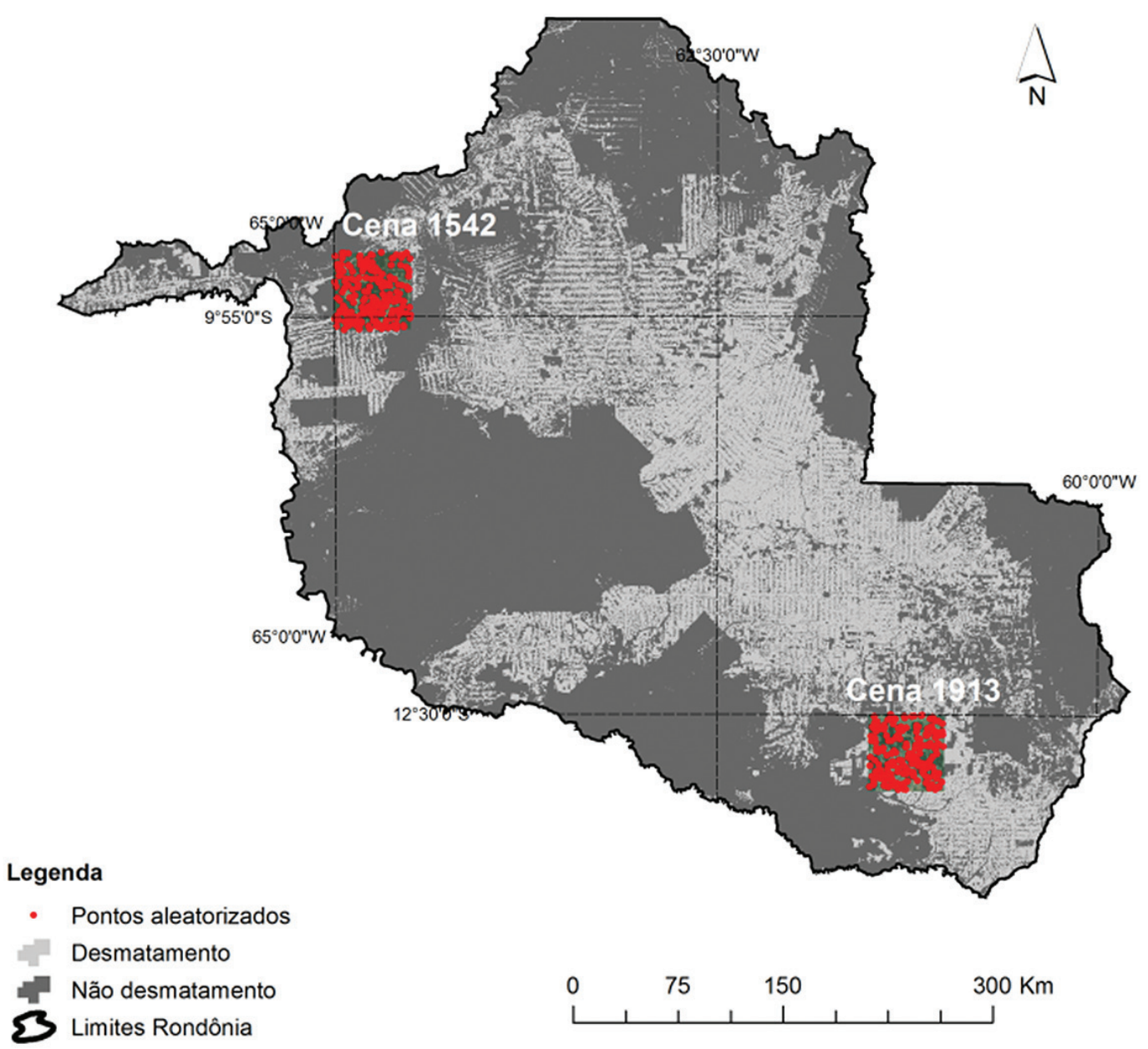

Figura 2. Localização das cenas do satélite SPOT utilizadas e os pontos aleatorizados (cor vermelha) para avaliação da acurácia das classificações do desmatamento no Estado de Rondônia.

Figure 2. SPOT scene locations and random sample points (red color) used for the accuracy assessment of Rondônia deforestation classifications.

$$
E G=\frac{T A}{n} \cdot 100
$$

em que: $\mathrm{EG}=$ exatidão global; TA = total de acertos; $\mathrm{n}=$ número total de pontos amostrados.

$$
k=\frac{N \sum_{i=1}^{r} x_{i i}-\sum_{i=1}^{r}\left(x_{i+} \cdot x_{+i}\right)}{N^{2}-\sum_{i=1}^{r}\left(x_{i+} \cdot x_{+i}\right)}
$$

em que: $\mathrm{K}=$ coeficiente Kappa; $\mathrm{N}=$ número total de pontos amostrados; $\mathrm{r}=$ número de linhas da matriz de erro; $x_{\mathrm{ii}=}$ valor na linha i e coluna $\mathrm{i} ; x_{+\mathrm{i}=}$ total da coluna $i ; x_{i+}=$ total da linha $i$.

Para avaliação do coeficiente Kappa, foi utilizada a Tabela 1.
Adicionalmente, as acurácias individuais de cada categoria (desmatado e não desmatado) foram determinadas, o que possibilitou a estimativa dos erros de inclusão (erros de comissão) (Equação 3) e os erros de exclusão (erros de omissão) (Equação 4).

$E c=\frac{x_{i+}-x_{i i}}{x_{i+}}$

$E o=\frac{x_{+i}-x_{i i}}{x_{+i}}$

O Erro de comissão (Ec) está relacionado com a exatidão do usuário, expressado por (1-Ec), que corresponde à confiabilidade que o usuário tem quanto aos resultados da classificação. Já o Erro de 
omissão (Eo) está relacionado com a exatidão do produtor, expressado por (1-Eo), em que o produtor conhece como ocorreu o acerto da classificação dos objetos.

\section{RESULTADOS E DISCUSSÃO}

Os resultados da acurácia dos mapeamentos estimados a partir da matriz de confusão dos mapeamentos do desmatamento conduzidos pelo PRODES e pela SEDAM, tomando em conta como verdade terrestre a interpretação visual das imagens SPOT do ano de 2010, são apresentados nas Tabelas 2 e 3 , respectivamente.

Os resultados da acurácia indicam que $\mathrm{O}$ mapeamento conduzido pela SEDAM apresentou uma acurácia global ligeiramente superior ao

Tabela 1. Classes do coeficiente Kappa.

Table 1. Kappa coefficient classes.

\section{Coeficiente Kappa}

$<0$

$0-0,2$

$0,21-0,4$

$0,41-0,6$

$0,61-0,8$

$0,81-1,0$

Fonte: Landis \& Koch (1977) mapeamento do PRODES-INPE, com acurácia de 94,8 e 93,3\%, respectivamente. Estes resultados revelam grande conformidade dos dados de detecção e estimativas do desmatamento produzidos pelo PRODES-INPE e pela SEDAM, com base em imagens LANDSAT 5 TM, quando comparados com imagens de referência (melhor resolução espacial) adquiridas pelo satélite SPOT.

Segundo a classificação do índice Kappa proposta por Landis \& Koch (1977) e com base nos resultados deste estudo, os mapeamentos do desmatamento produzidos pelo PRODES e SEDAM apresentaram Kappa superior a $81 \%$ e, portanto, são considerados excelentes. Do mesmo modo, considerando a classificação da acurácia global proposta por Foody (2002) e as acurácias globais observadas nesta análise - superiores a $85 \%$-, os mapeamentos do PRODES e SEDAM podem ser considerados de boa qualidade e há concordância das classes mapeadas com a realidade observada nas imagens de alta resolução do satélite SPOT 5.

Em relação à avaliação da acurácia de cada categoria (desmatado e não desmatado), a acurácia do produtor para a classe 'desmatamento' detectada pelo PRODES foi melhor do que a detectada pela SEDAM, sendo de 94,0\% e 93,0\%, respectivamente. Contudo, para a classe 'não desmatamento', os resultados foram inversos para os dois mapeamentos

Tabela 2. Matriz de confusão para classificação do desmatamento do PRODES.

Table 2. Confusion matrix for the PRODES deforestation classification.

\begin{tabular}{|c|c|c|c|c|c|}
\hline \multirow{2}{*}{\multicolumn{2}{|c|}{ Classe }} & \multicolumn{3}{|c|}{ Referência } & \multirow{2}{*}{$\begin{array}{c}\text { Erro de } \\
\text { comissão (\%) }\end{array}$} \\
\hline & & Desmatado & Não desmatado & $\Sigma$ & \\
\hline \multirow{4}{*}{$\begin{array}{c}\text { Dados de } \\
\text { Desmatamento }\end{array}$} & Desmatado & 187 & 15 & 202 & 7,4 \\
\hline & Não desmatado & 12 & 186 & 198 & 6,1 \\
\hline & $\Sigma$ & 199 & 201 & 400 & \multirow{2}{*}{6,7} \\
\hline & Erro de omissão (\%) & 6,0 & 7,5 & Erro Global (\%) & \\
\hline
\end{tabular}

Exatidão Global = 93,3\%; Coeficiente Kappa $=0,87$.

Tabela 3. Matriz de confusão do desmatamento da SEDAM.

Table 3. Confusion matrix for the SEDAM deforestation classification.

\begin{tabular}{|c|c|c|c|c|c|}
\hline \multirow{2}{*}{\multicolumn{2}{|c|}{ Classe }} & \multicolumn{3}{|c|}{ Referência } & \multirow{2}{*}{$\begin{array}{c}\text { Erro de } \\
\text { comissão (\%) }\end{array}$} \\
\hline & & Desmatado & Não desmatado & $\Sigma$ & \\
\hline \multirow{4}{*}{$\begin{array}{c}\text { Dados de } \\
\text { Desmatamento }\end{array}$} & Desmatado & 185 & 7 & 192 & 3,6 \\
\hline & Não desmatado & 14 & 194 & 208 & 6,7 \\
\hline & $\Sigma$ & 199 & 201 & 400 & \multirow{2}{*}{5,2} \\
\hline & Erro de omissão (\%) & 7,0 & 3,5 & Erro Global (\%) & \\
\hline
\end{tabular}

Exatidão Global = 94,8\%; Coeficiente Kappa $=0,89$. 
avaliados, com acurácia do produtor de 92,5\% e 96,5\% para o PRODES e SEDAM, respectivamente.

As acurácias do usuário foram determinadas como sendo de $96,4 \%$ e $92,6 \%$ para a classe 'desmatamento', detectadas pela SEDAM e pelo PRODES-INPE, respectivamente. Tais resultados revelam uma estimativa mais conservadora para a classe 'desmatamento' da SEDAM, que apresentou menor superestimação desta classe, além de melhor acurácia, de forma geral.

Com base nos resultados desta análise, apesar de utilizarem diferentes metodologias, as diferenças entre as acurácias dos mapeamentos da SEDAM e do PRODES foram semelhantes. Tal fato é muito relevante, pois os resultados do PRODES são baseados em metodologia semiautomática, o que dá maior rapidez ao processo de classificação e obtenção dos resultados do desmatamento. Por outro lado, a SEDAM implementa o mapeamento do desmatamento, tendo como base técnica a interpretação visual que, além de tomar mais tempo, é intérprete-dependente.

Em relação aos resultados do desmatamento, o PRODES estima um total desmatado 5\% superior ao estimado pela SEDAM em todo o Estado de Rondônia até 2011. Assim, enquanto o PRODES estimou 8.070.300 hectares, a SEDAM estimou 7.671.700 hectares desmatados (Figura 3).

Os anos mais críticos de desmatamento no período estudado foram observados entre 2002 e 2005, com totais anuais desmatados sempre superiores a 300.000 hectares, estimados por pelo menos um dos programas de mapeamento estudado. Essas altas taxas de desmatamento podem estar relacionadas ao alto crescimento econômico da região no período em questão. Neste sentido, apenas os Estados do Pará, do Amazonas e de Rondônia, da Região Norte, tiveram crescimento positivo de $0,1 \%$ da participação nacional do PIB do Brasil entre 2002 e 2005. Além disso, o Estado de Rondônia apresentou um crescimento de $20 \%$ do Produto Interno Bruto (PIB) per capita entre 2002 e 2005, bem próximo da média da Região Norte e duas vezes maior do que o restante do país, que apresentaram, no mesmo período, um crescimento de $21,7 \%$ e $10,0 \%$ deste mesmo indicador de crescimento econômico, respectivamente (IBGE, 2007).

E, de fato, investimentos governamentais em programas de desenvolvimento (Pfaff, 1999; Kirby et al., 2006), linhas de créditos subsidiadas (Andersen \& Reis, 1997), construção e pavimentação de estradas e outros investimentos em infraestrutura contribuem para o crescimento econômico da região e, consequentemente, para o aumento da probabilidade de uma área de floresta ser desmatada na Amazônia brasileira (Andersen \& Reis, 1997; Kirby et al., 2006).

Enquanto isso, embora o PRODES tenha estimado maior área total desmatada até 2011, a SEDAM estimou maiores taxas anuais de desmatamento, exceto para os anos de 2004 e 2005. Assim, é possível

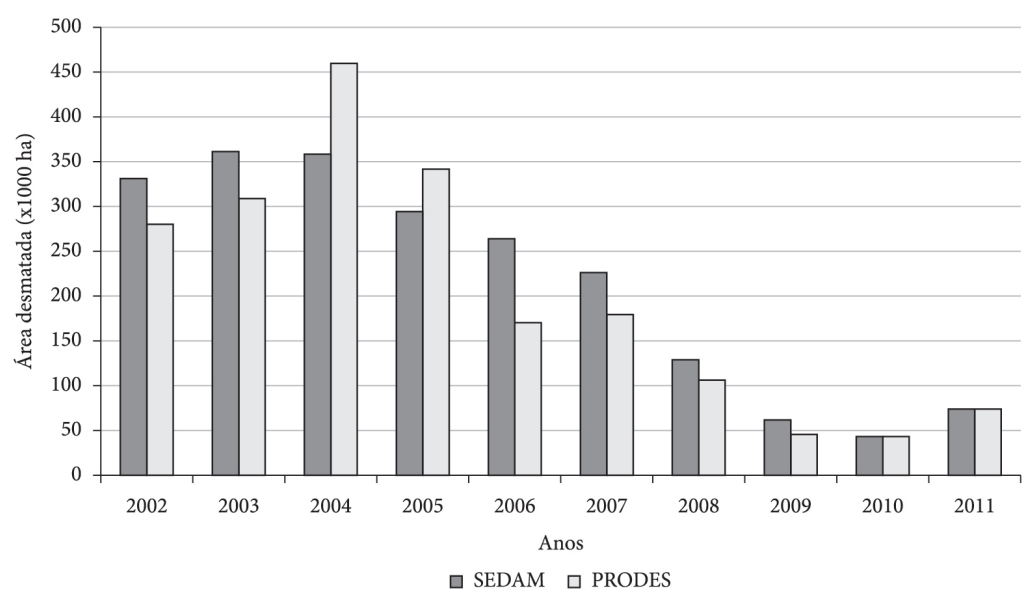

Figura 3. Desmatamento anual estimado pela SEDAM e o PRODES entre 2002 e 2011.

Figure 3. Annual deforestation estimated by SEDAM and PRODES between 2002 and 2011. 


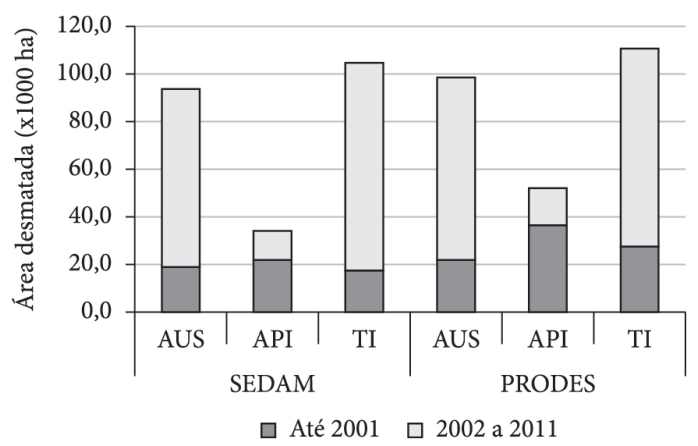

Figura 4. Desmatamento nas áreas protegidas detectado pela SEDAM e PRODES.

Figure 4. Deforestation within protected areas detected by SEDAM and PRODES.

inferir que as áreas desmatadas quantificadas pelo PRODES, responsáveis pelo total desmatado até 2011, foram detectadas em mapeamentos realizados em períodos anteriores ao ano de 2002.

Por outro lado, o mapeamento realizado pelo PRODES estimou a maior área desmatada no interior das áreas protegidas (TI, API e AUS) existentes em Rondônia. Os resultados revelam ainda que a maioria dos desmatamentos dentro das áreas protegidas, exceto nas API, ocorreu depois do ano de 2001 (Figura 4).

Estes resultados são significativos, pois apresentam um crescimento substancial do desmatamento dentro de Unidades de Conservação de Uso Sustentável e Terras Indígenas, na última década. Este fato já havia sido alertado por Pedlowski et al. (2005), que observaram que várias unidades de conservação em Rondônia estavam sob forte ameaça, fruto da presença de atividades de extração ilegal de madeira e agropecuária. Pedlowski e seus colaboradores também apontaram que a Floresta Nacional do Bom Futuro, em Rondônia, apresentava uma forte tendência de desmatamento ilegal e, se tudo continuasse naquele cenário, não haveria mais floresta nesta área (des)protegida, até o ano de 2017. Essas tendências foram consolidadas mais recentemente pela Lei Federal n. ${ }^{\circ} 12.249$, de 11 de junho de 2010 (Brasil, 2010), que alterou os limites e reduziu a área da Floresta Nacional do Bom Futuro em cerca de 190.000 hectares, correspondentes a mais de $65 \%$ de toda sua área original.
Finalmente, é importante apontar que, além do desmatamento analisado neste estudo, a exploração seletiva de madeira e os incêndios florestais também afetam a integridade ecológica das florestas tropicais nativas. Estas áreas de florestas impactadas pelo fogo e por atividades madeireiras podem alcançar dimensões semelhantes às áreas desmatadas na região Amazônica (Nepstad et al., 1999; Asner et al., 2005; Matricardi et al., 2010, 2013).

\section{CONCLUSÕES}

O mapeamento conduzido pela SEDAM apresentou melhores resultados de acurácia global e coeficiente Kappa do que o conduzido pelo PRODES. Se as classes de uso estudadas ('desmatadas' e 'não desmatadas') forem consideradas, o mapeamento da SEDAM se mostrou mais conservador na estimativa do desmatamento, pois apresentou menor erro de superestimação e maior erro de subestimação, quando comparado com os resultados do PRODES. As diferenças entre as acurácias dos mapeamentos do desmatamento conduzidos pela SEDAM e pelo PRODES podem ser apontadas como inexpressivas, podendo ambas ser consideradas muito boas. Entretanto, a diferença substancial está nas metodologias utilizadas pela SEDAM e pelo PRODES, baseadas na interpretação visual e na classificação semiautomática, respectivamente. Este fato implica numa maior rapidez de respostas das estimativas do desmatamento pelo programa de mapeamento do PRODES, com acurácia semelhante, quando comparado com o programa da SEDAM. Note-se que este é um fator de grande relevância, em se tratando de monitoramento de processos de destruição ambiental na Amazônia.

Uma questão importante, que foi demonstrada pelos resultados de ambos os mapeamentos (PRODES e SEDAM), foi o aumento substancial do desmatamento ilegal ocorrido nos últimos dez anos dentro de áreas protegidas (incluindo terras indígenas e unidades de conservação de uso direto) no Estado de Rondônia. Neste caso, o desmatamento aumentou 400\% entre 2002 e 2011 em relação ao total desmatado até 2001, dentro de áreas protegidas em Rondônia. Se esta tendência persistir, estas áreas estarão sob sério risco de descaracterização e degradação, ou mesmo de completa destruição. 


\section{AGRADECIMENTOS}

Agradecemos à Administração e ao Pessoal Técnico da SEDAM-RO, pelo apoio durante os trabalhos de campo e pelo fornecimento de dados.

\section{STATUS DA SUBMISSÃO}

Recebido: 27 nov., 2013

Aceito: 10 mar., 2014

Publicado: 28 ago., 2014

\section{AUTOR(ES) PARA CORRESPONDÊNCIA}

\section{Eraldo Aparecido Trondoli Matricardi}

Departamento de Engenharia Florestal,

Universidade de Brasília - UnB, CEP 70910-900,

Brasília, DF, Brasil

e-mail: ematricardi@unb.br

\section{REFERÊNCIAS}

Andersen LE, Reis EJ. Deforestation, Development, and Government Policy in the Brazilian Amazon: An Econometric Analysis. Rio de Janeiro: IPEA; 1997.

Asner GP, Knapp DE, Broadbent EN, Oliveira PJC, Keller M, Silva JN. Selective Logging in the Brazilian Amazon. Science 2005; 310(5747): 480-482. PMid:16239474. http://dx.doi.org/10.1126/science.1118051

Becker FG. Aplicações de Sistemas de Informação Geográfica em Ecologia e Manejo de Bacias Hidrográficas. In: Schiavetti A, organizador. Bacias Hidrográficas: Teorias e Aplicações. Bahia: Editus; 2002. p. 91-111.

Brandão A Jr, Souza C Jr. Desmatamento nos Assentamentos de Reforma Agrária na Amazônia. O Estado da Amazônia. Belém: Imazon; 2006.

Brasil. Departamento Nacional de Produção Mineral. Projeto RADAMBRASIL. Levantamento de recursos naturais. Rio de Janeiro; 1978.

Brasil. Lei $n^{\circ} 12.249$, de 11 de junho de 2010. Diário Oficial da República Federativa do Brasil, Brasília, DF (2010 jun. 14). Seção 1, p. 1677-7042.

Câmara G, Valeriano DM, Soares JV. Metodologia para o cálculo da taxa anual de desmatamento na Amazônia Legal. São José dos Campos: INPE; 2006 [cited 2013 Oct 28]. Available from: http://www.obt.inpe.br/prodes/ metodologia.pdf.

Congalton R, Mead R. A Review of Three Discrete Multivariate Analysis Techniques Used in Assessing the Accuracy of Remotely Sensed Data from Error Matrices.
IEEE Transactions on Geoscience and Remote Sensing 1986; GE-24(1): 169-174 [cited 2014 Jan 29]. Available from: http://ieeexplore.ieee.org.

Foody GM. Status of land cover classification accuracy assessment. Remote Sensing of Environment 2002; 80(1): 185-201. http://dx.doi.org/10.1016/S00344257(01)00295-4

Gasparini KAC, Lyra GB, Francelino MR, Delgado RC, Oliveira JFO Jr, Facco AG. Técnicas de geoprocessamento e sensoriamento remoto aplicadas na identificação de conflitos do uso da terra em Seropédica - RJ. Floresta e Ambiente 2013; 20(3):296-306.

Instituto Brasileiro de Geografia e Estatística - IBGE. Contas Regionais do Brasil 2002 - 2005. 2007 [cited 2014 Jan 29]. Available from: http://www.ibge.gov.br/home/ estatistica/economia/contasregionais/2002_2005/ default.shtm.

Kirby KR, Laurance WF, Albernaz AK, Schoroth G, Fearnside PM, Bergen S, et al. The future of deforestation in the Brazilian Amazon. Futures 2006; 38: 432-453.. http://dx.doi.org/10.1016/j.futures.2005.07.011

Landis J, Koch GG. The measurements of observer agreement for categorical data. Biometrics 1977; 33(3): 159-179. PMid:843571. http://dx.doi. org/10.2307/2529310

Lingnau C, Antunes AFB. Avaliação da Acurácia de Mapas Temáticos Oriundos de Classificação Digital de Imagens. SIMGEO, 2003 [cited 2013 Sept 21]. Available from: http://www.cieg.ufpr.br.

Matricardi EAT, Skole DL, Pedlowski MA, Chomentowski W, Fernandes LC. Assessment of tropical forest degradation by selective logging and fire using Landsat imagery. Remote Sensing of Environment 2010; 114: 1117-1129. http://dx.doi.org/10.1016/j. rse.2010.01.001

Matricardi EAT, Skole DL, Pedlowski MA, Chomentowski W. Assessment of forest disturbances by selective logging and forest fires in the Brazilian Amazon using Landsat data. International Journal of Remote Sensing. 2013; 34: 1057-1086. http://dx.doi.org /10.1080/01431161.2012.717182

Nepstad DC, Verssimo A, Alencar A, Nobre C, Lima E, Lefebvre P, et al. Large-scale impoverishment of Amazonian forests by logging and fire. Nature 1999; 398(6727): 505-508. http://dx.doi.org/10.1038/19066

Pfaff ASP. What Drives Deforestation in the Brazilian Amazon? Journal of Environmental Economics and Management 1999; 37: 26-43. http://dx.doi.org/10.1006/ jeem.1998.1056

Pedlowski MA, Matricardi EAT, Skole D, Cameron SR, Chomentowski W, Fernandes LC, et al. Conservation units: a new deforestation frontier in the Amazonian state of Rondônia, Brazil. Environmental Conservation 
2005; 32(2): 149-155. http://dx.doi.org/10.1017/ S0376892905002134

Powell RL, Matzke N, Souza C Jr, Clark M, Numata I, Hess LL, et al. Sources of error in accuracy assessment of thematic land-cover maps in the Brazilian Amazon. Remote Sensing of Environment 2004; 90: 221-234. http://dx.doi.org/10.1016/j.rse.2003.12.007

Rondônia. Governo do Estado. Zoneamento Socioeconômico-Ecológico do Estado de Rondônia - ZSEE: Um Instrumento de Gestão Ambiental a Serviço do Desenvolvimento Sustentável de Rondônia. Porto Velho: SEDAM; 2007.
Shimabukuro YE, Smith JA. The least-squares mixing models do generate fraction images derived from remote sensing multispectral data. IEEE Transactions on Geosciences and Remote Sensing 1991; 29(1): 16-20. http://dx.doi.org/10.1109/36.103288

Thapa K, Bossler DJ. Accuracy of Spatial Data Used in Geographic Information Systems. Photogrammetric Engineering and Remote Sensing 1992; 58(6): 835-841.

Tourneau FML, Bursztyn M. Assentamentos rurais na Amazônia: contradições entre a política agrária e a política ambiental. Ambiente \& Sociedade 2010; 13(1): 111-130. http://dx.doi.org/10.1590/S1414753X2010000100008 